\title{
Applicability of HIN-1, MGMT and RASSF1A promoter methylation as biomarkers for detecting field cancerization in breast cancer
}

\author{
Melanie Spitzwieser ${ }^{1}$, Elisabeth Holzweber ${ }^{1}$, Georg Pfeiler ${ }^{2}$, Stefan Hacker ${ }^{3}$ and Margit Cichna-Markl ${ }^{*}$
}

\begin{abstract}
Introduction: It has been shown in some articles that genetic and epigenetic abnormalities cannot only be found in tumor tissues but also in adjacent regions that appear histologically normal. This phenomenon is metaphorically called field cancerization or field defect. Field cancerization is regarded as clinically significant because it is assumed to be an important factor in local recurrence of cancer. As the field showing these molecular abnormalities may not be removed completely by surgery, these changes might lead to neoplasms and subsequent transformation to a tumor. We aimed to investigate the applicability of the methylation status of six tumor suppressor genes as biomarkers for detecting field cancerization in breast cancer.
\end{abstract}

Methods: The promoter methylation status of CCND2, DAPK1, GSTP1, HIN-1, MGMT and RASSF1A was determined by methylation-sensitive high-resolution melting (MS-HRM) analysis. MS-HRM methods for CCND2, MGMT and RASSF1A were developed in-house, primer sequences for DAPK1, GSTP1 and HIN-1 have already been published. Biopsy samples were taken from tumor, tumor-adjacent and tumor-distant tissue from 17 breast cancer patients. Normal breast tissues of four healthy women served as controls.

Results: All MS-HRM methods proved to be very sensitive. LODs were in the range from 0.1 to $1.5 \%$, LOQs ranged from 0.3 to $5.3 \%$. A total of $94 \%, 82 \%$ and $65 \%$ of the tumors showed methylation of RASSFIA, HIN-1 and MGMT promoters, respectively. The methylation status of these promoters was significantly lower in tumor-distant tissues than in tumor tissues. Tumor-adjacent tissues showed higher methylation status of RASSF1A, HIN-1 and MGMT promoters than tumor-distant tissues, indicating field cancerization. The methylation status of the HIN-1 promoter in tumor-adjacent tissues was found to correlate strongly with that in the corresponding tumors $(r=0.785, p<0.001)$, but not with that in the corresponding tumor-distant tissues $(r=0.312, p=0.239)$.

Conclusions: Among the gene promoters investigated, the methylation status of the HIN-1 promoter can be considered the best suitable biomarker for detecting field cancerization. Further investigation is needed to test whether it can be used for defining surgical margins in order to prevent future recurrence of breast cancer.

\section{Introduction}

Cancer can be considered as cumulative phenotypic consequence of acquired genetic and epigenetic alterations in cells [1]. Epigenetic alterations, in particularly changes in the DNA methylation pattern, are known to play a crucial role in carcinogenesis. Aberrant DNA methylation occurs early in carcinogenesis, suggesting that DNA methylation alterations may precede classical

\footnotetext{
* Correspondence: margit.cichna@univie.ac.at

'Department of Analytical Chemistry, University of Vienna, Währinger Str. 38, 1090 Vienna, Austria

Full list of author information is available at the end of the article
}

transforming events such as gene mutations. Changes in the DNA methylation status occur more frequently than mutations or cytogenic abnormalities [2].

In humans and other mammals, DNA methylation takes place at carbon-5 of cytosine residues within cytosine-phosphatidyl-guanosine (CpG) dinucleotides. CpG dinucleotides are heterogeneously distributed in the human genome, often clustered in so-called CpG islands. $\mathrm{CpG}$ islands are particularly present in promoter regions and first exons of genes that regulate important cell functions [3]. 
In normal cells, CpG islands are generally unmethylated, resulting in gene expression, if the corresponding transcription factors are available [2]. In cancer cells, however, the promoter region of certain genes is frequently hypermethylated, leading to a tightly packed chromatin and transcriptional gene silencing. Promoter hypermethylation commonly affects regulator genes that are involved in a wide range of cellular pathways, such as cell cycle, DNA repair, toxic catabolism, cell adherence, apoptosis and angiogenesis [4].

Several studies have already investigated the applicability of promoter hypermethylation as specific and sensitive biomarkers, e.g., for the detection and diagnosis of cancer at an early stage $[5,6]$, the prognosis of cancer $[7,8]$ or the prediction of the response to a certain treatment scheme $[9,10]$. In contrast to genetic alterations, changes in the DNA methylation status are potentially reversible. Reactivation of epigenetically silenced genes by using DNA demethylating drugs is therefore regarded as a promising strategy in cancer therapy $[11,12]$.

Recent studies have shown that molecular abnormalities occur not only in the tumor tissue but also in tissue that is adjacent to the tumor and appears histologically normal. The presence of such abnormalities in tissues surrounding tumors is called field cancerization or field defect [13]. In addition to genetic abnormalities, e.g., chromosomal anomalies and loss of heterozygosity, epigenetic alterations, in particular changes in the DNA methylation status, have been found in normalappearing tissues close to tumors. Molecular signatures of field cancerization have been reported for various epithelial tumors including those of the colon [14-16] and the prostate $[17,18]$.

Field cancerization is of clinical relevance because it is assumed to be an important factor in local recurrence of cancer [19]. As the field showing aberrant DNA methylation may not be removed completely by surgery, these changes in the DNA methylation status might lead to neoplasms and subsequent transformation to a tumor. So far, only a limited number of studies have investigated changes in the DNA methylation status in histologically normal tissue adjacent to breast tumor tissues. Yan et al. [20] detected methylation changes in the promoter of Ras association domain family member 1 (RASSF1A) in mammary tissue as far as $4 \mathrm{~cm}$ from the primary tumor. In a study of Feng et al. [21] the DNA methylation status of reversion-induced LIM protein (RIL), high in normal1 (HIN-1), RASSF1A and cadherin-13 (CDH13) in normal-appearing tissue (located at least $3 \mathrm{~cm}$ away from the tumor) was found to correlate with that in the breast tumor.

The main aim of the present study was to extend research on the applicability of the methylation status of candidate genes as biomarkers for field cancerization.
We selected a panel of six tumor suppressor genes that have previously been reported to be frequently methylated in breast tumors, comprising cyclin D2 (CCND2), death-associated protein kinase 1 (DAPK1), glutathione S-transferase P1 (GSTP1), HIN-1, O6-methylguanineDNA methyltransferase (MGMT) and RASSF1A [22]. From each of 17 breast cancer patients, three biopsy samples were taken: the first one from the tumor tissue, the second one from tumor-adjacent tissue and the third one from tumor-distant tissue. In addition, we tested if the DNA methylation status of the six tumor suppressor genes in tumor, tumor-adjacent and/or tumor-distant tissues is associated with any clinicopathological parameters. We were also interested to see if there is a correlation between the DNA methylation status of the genes in the tissues of one and the same breast cancer patient.

\section{Methods}

\section{Patients and biopsy samples}

The study was approved by the Ethics Commission of the Medical University of Vienna (application number 1074/2011). All patients gave written informed consent. Biopsy samples from 17 breast cancer patients (aged 3976 years, mean age: 58 years) were taken by ultrasoundguided needle biopsies. None of the patients had a family history of breast cancer. From each patient, three biopsy samples were taken: the first one directly from the primary breast tumor, the second one from histologically normal tissue located about $1 \mathrm{~cm}$ from the tumor ("tumor-adjacent tissue") and the third one from histologically normal tissue located about $3 \mathrm{~cm}$ away from the tumor ("tumor-distant tissue"). In addition, breast tissue samples were obtained from four women (aged 44-60 years, mean age: 53 years) undergoing breast reduction mammoplasty. From two of these women, samples were obtained from both the left and right breast.

All biopsy samples were stored in phosphate-buffered saline (PBS) at $-80{ }^{\circ} \mathrm{C}$ until analysis.

\section{Patient characteristics}

Patient characteristics including age, menopausal status, histologic type, histological grading, B classification, proliferative activity (MIB-1), status of estrogen receptor (ER), progesterone receptor (PR) and human epidermal growth factor receptor 2 (HER2) as well as the molecular subtype are summarized in Table 1. Information on menopausal status, histological grading, MIB-1 and the receptor status was, however, lacking for one, two, one and one patients, respectively.

\section{Extraction of genomic DNA}

Genomic DNA was extracted from biopsy samples using the QIAamp DNA Mini Kit (Qiagen, Hilden, Germany) 
Table 1 Characteristics of breast cancer patients

\begin{tabular}{|c|c|c|c|c|c|c|c|c|c|c|}
\hline \multirow[t]{2}{*}{ Patient } & \multirow[t]{2}{*}{ Age (y) } & \multirow[t]{2}{*}{ Menopause status } & \multirow[t]{2}{*}{ Histologic type } & \multirow[t]{2}{*}{ Histological grading } & \multirow[t]{2}{*}{ B classification } & \multirow[t]{2}{*}{ MIB-1 (\%) } & \multicolumn{3}{|c|}{ Receptor status } & \multirow[t]{2}{*}{ Molecular subtype } \\
\hline & & & & & & & $\overline{E R}$ & $P R$ & HER2 & \\
\hline 1 & 75 & Post & IDC & 2 & $5 b$ & 10 & + & + & - & Luminal A \\
\hline 2 & 65 & Post & IDC & 2 & $5 b$ & 10 & + & + & - & Luminal A \\
\hline 3 & 54 & n.s. & IDC & 3 & $5 b$ & n.s. & n.s. & n.s. & n.s. & n.s. \\
\hline 4 & 39 & Pre & IDC & 2 & $5 b$ & 40 & + & + & + & Luminal B \\
\hline 5 & 66 & Post & IDC & 2 & $5 b$ & 60 & + & + & - & Luminal A \\
\hline 6 & 50 & Pre & IDC & 3 & $5 b$ & 50 & + & + & + & Luminal B \\
\hline 7 & 73 & Post & IDC & 3 & $5 b$ & 20 & + & + & - & Luminal A \\
\hline 8 & 76 & Post & IDC & 2 & $5 b$ & 20 & + & + & - & Luminal A \\
\hline 9 & 63 & Post & IDC & 3 & 5 & 30 & + & + & - & Luminal A \\
\hline 10 & 48 & Post & IDC & 3 & $5 b$ & 20 & + & + & + & Luminal B \\
\hline 11 & 58 & Post & IDC & n.s. & $5 c$ & 20 & + & + & + & Luminal B \\
\hline 12 & 61 & Post & IDC & 3 & $5 b$ & 70 & - & - & - & Triple negative \\
\hline 13 & 52 & Pre & ILC & n.s. & $5 b$ & 50 & + & + & - & Luminal A \\
\hline 14 & 42 & Pre & IDC & 3 & $5 b$ & 80 & + & - & - & Luminal A \\
\hline 15 & 67 & Post & IDC & 3 & $5 b$ & 40 & + & + & - & Luminal A \\
\hline 16 & 61 & Post & ILC & 2 & $5 b$ & 30 & + & + & - & Luminal A \\
\hline 17 & 41 & Pre & Mucinous & 2 & $5 b$ & 50 & + & + & + & Luminal B \\
\hline
\end{tabular}

MIB-1 mindbomb E3 ubiquitin protein ligase 1, ER estrogen receptor, PR progesterone receptor, HER2 human epidermal growth factor receptor 2, IDC invasive ductal carcinoma, ILC invasive lobular carcinoma, n.s. not specified

according to the manufacturer's protocol. The DNA concentration was determined using a NanoDrop 2000c spectrophotometer (Thermo Fisher Scientific, Waltham, MA, USA).

\section{Methylation-sensitive high-resolution melting (MS-HRM) analysis}

DNA extracted from biopsy samples and human control DNA (fully methylated and unmethylated) were treated with sodium bisulfite using the EpiTect Fast DNA Bisulfite Kit (Qiagen) according to the manufacturer's instructions. Fully methylated control DNA (CpGenom Universal Methylated DNA) was obtained from EMD Millipore (Billerica, MA, USA), unmethylated control DNA (EpiTect Control DNA (human), unmethylated) from Qiagen.

Primers for CCND2 (GenBank: CM000263.1), MGMT (GenBank: X61657.1) and RASSF1A (GenBank: NG_023270.1), targeting CpG island regions flanking the transcription site, were designed with the Methyl Primer Express Software v1.0 (Applied Biosystems, Carlsbad, CA, USA). Primer sequences for DAPK1 [23], GSTP1 [24] and HIN-1 [25] were taken from the literature. For each MS-HRM method, the annealing temperature $\left(\mathrm{T}_{\mathrm{a}}\right)$ and the $\mathrm{MgCl}_{2}$ concentration were optimized in-house. Primer sequences and optimized conditions are summarized in Table 2.
Polymerase chain reaction (PCR) amplification of the bisulfite-treated DNA and HRM analysis were performed using a Rotor-Gene Q thermocycler (Qiagen). Each reaction mixture had a total volume of $20 \mu \mathrm{l}$, containing $10 \mathrm{ng}$ of bisulfite-treated DNA, $10 \mu \mathrm{l}$ of $2 \times$ EpiTect HRM PCR Master Mix (Qiagen), forward and reverse primer and RNase-free water. In all PCR reactions, the concentration of forward and reverse primer was $250 \mathrm{nM}$ each. PCR amplification was carried out under the following conditions: initial step at $95{ }^{\circ} \mathrm{C}$ for $5 \mathrm{~min}$; followed by 50 cycles at $95{ }^{\circ} \mathrm{C}$ for $10 \mathrm{~s}, \mathrm{~T}_{\mathrm{a}}$ of the respective primer set for $30 \mathrm{~s}$ and $72{ }^{\circ} \mathrm{C}$ for $10 \mathrm{~s}$ (touchdown $1{ }^{\circ} \mathrm{C}, 7$ cycles); denaturation step at $95{ }^{\circ} \mathrm{C}$ for $1 \mathrm{~min}$ followed by a hybridization step at $40{ }^{\circ} \mathrm{C}$ for $1 \mathrm{~min}$. In the HRM step, the temperature was increased by $0.1{ }^{\circ} \mathrm{C}$ increments per $2 \mathrm{~s}$.

HRM data were evaluated with the Rotor-Gene Q Series Software 2.1.0 (Qiagen). Each biopsy sample was analyzed at least twice in duplicate. The DNA methylation status was calculated with the help of calibration curves established by analyzing mixtures of fully methylated and unmethylated human control DNA. In order to obtain accurate results also for heterogeneously methylated sequences, an interpolation calibration curve was established as proposed by Migheli et al. [26]. However, we slightly changed their approach and calculated the average of the normalized fluorescence signal for each 
Table 2 Primer sequences and methylation-sensitive high-resolution melting (MS-HRM) conditions

\begin{tabular}{|c|c|c|c|c|c|c|c|}
\hline Gene & Primer sequences & $\begin{array}{l}\text { Additional } \mathrm{MgCl}_{2} \\
\text { concentration (mM) }\end{array}$ & $\mathrm{T}_{\mathrm{a}}\left({ }^{\circ} \mathrm{C}\right)$ & Amplicon length (bp) & $\begin{array}{l}\text { Number of } \\
\text { CpGs }\end{array}$ & LOD / LOQ (\%) & Reference \\
\hline \multirow[t]{2}{*}{ CCND2 } & F: 5' GTTTAGAGCGGAGAAGAG 3' & 0 & 50 & 89 & 4 & $0.1 / 0.3$ & In-house \\
\hline & R: 5' AACAAAACCTCGAAACTACC 3' & & & & & & \\
\hline \multirow[t]{2}{*}{ DAPK1 } & F: 5' GCGCGGAGTTGGGAGGAG 3' & 0 & 57 & 70 & 7 & $0.2 / 0.9$ & [23] \\
\hline & R: 5' CTCCGAACTACCCTACCAAACC 3' & & & & & & \\
\hline \multirow[t]{2}{*}{ GSTP1 } & F: 5' GTGAAGCGGGTGTGTAAGTTT 3' & 1 & 56 & 120 & 12 & $0.9 / 3.3$ & [24] \\
\hline & R: 5' TAAACAAACAACAAAAAAAAAACC 3' & & & & & & \\
\hline \multirow[t]{2}{*}{$H I N-1$} & F: 5' GCGAGGATCGGGTATAAGAAGTT 3' & 2 & 55 & 133 & 12 & $0.3 / 1.4$ & {$[25]$} \\
\hline & R: 5' CACCGAAACATACAAAACAAAACCA 3' & & & & & & \\
\hline \multirow[t]{2}{*}{ MGMT } & F: 5' TTGATTAGGGGAGCGGTATTAG 3' & 2 & 52 & 140 & 14 & $0.9 / 3.0$ & In-house \\
\hline & R: 5' CCACATACCCGAATAATCCTAAAA 3' & & & & & & \\
\hline \multirow[t]{2}{*}{ RASSFIA } & F: 5' GTCGGGGTTTGTTTTGTGGTT 3' & 2 & 56 & 118 & 9 & $1.5 / 5.3$ & In-house \\
\hline & R: 5' CAACTCCCACAACTCAATAAACT 3' & & & & & & \\
\hline
\end{tabular}

$T_{a}$ annealing temperature, $b p$ base pair, $C p G$ cytosine-phosphatidyl-guanosine, $L O D$ limit of detection, $L O Q$ limit of quantification

standard over the entire temperature interval instead of using single values at chosen temperature points. Calibration functions were established with SigmaPlot 11.0 (Systat Software Inc., San Jose, CA, USA). Limit of detection (LOD) and limit of quantification (LOQ) of the MS-HRM methods were determined by repeatedly analyzing bisulfite-treated, unmethylated control DNA. After calculating the mean and the standard deviation, the LOD (signal-to-noise ratio $(\mathrm{S} / \mathrm{N})$ of 3 ) was determined by adding three times the standard deviation and the LOQ $(\mathrm{S} / \mathrm{N}$ of 10$)$ by adding ten times the standard deviation to the mean. The corresponding methylation status was then calculated with the help of the equation of the calibration curves established by analyzing mixtures of methylated and unmethylated control DNA.

\section{Statistical analysis}

Statistical analyses were carried out with IBM SPSS Statistics 21.0 (IBM Corp., Armonk, NY, USA). The methylation status was treated either as categorical variable (< LOD, < LOQ or $\geq$ LOQ) or as continuous variable. If the methylation status was treated as continuous variable, methylation status $\angle \mathrm{LOD}$ and $<\mathrm{LOQ}$ were substituted with a default value, namely half the LOD and half the LOQ, respectively, as proposed previously [27]. Chi-squared test was used to evaluate if the methylation status of the six genes is associated with any of the clinicopathological parameters. Independent-samples $t$ test was applied to evaluate if there are significant differences in the methylation status between the tumor tissues and noncancerous breast tissues of the control group. One-way ANOVA (analysis of variance), followed by Tukey's test, was applied to test for significant differences in the DNA methylation status between tumor, tumor-adjacent and tumor-distant tissues. Levene's test was used to assess the equality of variances. Pearson's correlation coefficient was used to assess the relationship between two continuous variables. In all cases, $p<0.05$ (two-sided) was considered significant.

\section{Results}

\section{Validation of the MS-HRM methods}

MS-HRM methods for CCND2, MGMT and RASSF1A were developed in-house, primer sequences for DAPK1, GSTP1 and HIN-1 were taken from previously published articles. After optimizing the $\mathrm{T}_{\mathrm{a}}$ and the $\mathrm{MgCl}_{2}$ concentration added to the commercial HRM PCR Master Mix (Table 2), the methods were validated with regard to limit of detection (LOD), limit of quantification (LOQ) and inter-day repeatability. LOD and LOQ of the MS-HRM methods were determined by repeatedly analyzing bisulfite-treated, unmethylated control DNA. LODs $(\mathrm{S} / \mathrm{N}=3)$ and LOQs $(\mathrm{S} / \mathrm{N}=10)$ were in the range from 0.1 to $1.5 \%$ and 0.3 to $5.3 \%$, respectively (Table 2). These data demonstrate that the MS-HRM methods are applicable to detect and quantify low methylation levels. The repeatability of the methods was investigated by repeatedly analyzing mixtures of unmethylated and fully methylated control DNA. Figure 1a shows normalized melting curves of mixtures of unmethylated and fully methylated control DNA for DAPK1. The representative calibration curve, obtained by analyzing mixtures of unmethylated and fully methylated control DNA on 4 days in duplicate, indicates the high inter-day repeatability of the method (Fig. 1b).

\section{Heterogeneous methylation}

MS-HRM methods make it possible to determine the methylation status across all $\mathrm{CpG}$ dinucleotides within the amplicon, but they do not allow determining the 

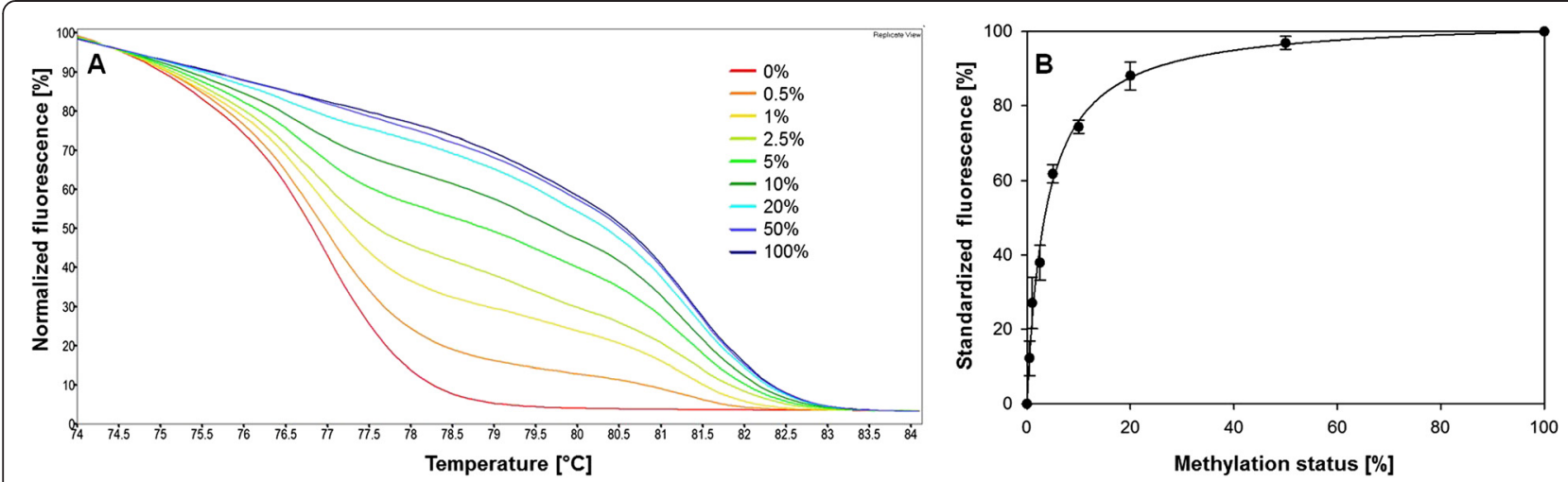

Fig. 1 Repeatability of the high-resolution melting (HRM) method for DAPK1. a Normalized melting curves of mixtures of unmethylated and fully methylated control DNA. Methylation status of the standards: $0 \%, 0.5 \%, 1 \%, 2.5 \%, 5 \%, 10 \%, 20 \% 50 \%, 100 \%$. Replicate view of duplicate measurements carried out on 1 day. $\mathbf{b}$ Calibration curve obtained by analyzing mixtures of unmethylated and fully methylated control DNA in duplicate on 4 different days $(n=8)$

methylation status of individual CpG dinucleotides. However, in contrast to several other methods, MSHRM analysis is applicable to detect heterogeneous methylation [28-30]. Melting curves obtained for heterogeneously methylated templates differ in the shape from those obtained for mixtures of unmethylated and fully methylated control DNA. In addition, the melt profile derivative plots do not contain distinct peaks as observed for unmethylated and fully methylated DNA (Fig. 2). When we applied the MSHRM methods to biopsy samples of breast cancer patients, the promoters of CCND2, GSTP1, HIN-1 and RASSF1A were generally found to be methylated homogeneously. Melting profiles obtained for MGMT and DAPK1 indicated that most biopsy samples showed homogeneous methylation whereas in some samples, MGMT and/or DAPK1 were methylated heterogeneously (Fig. 2).

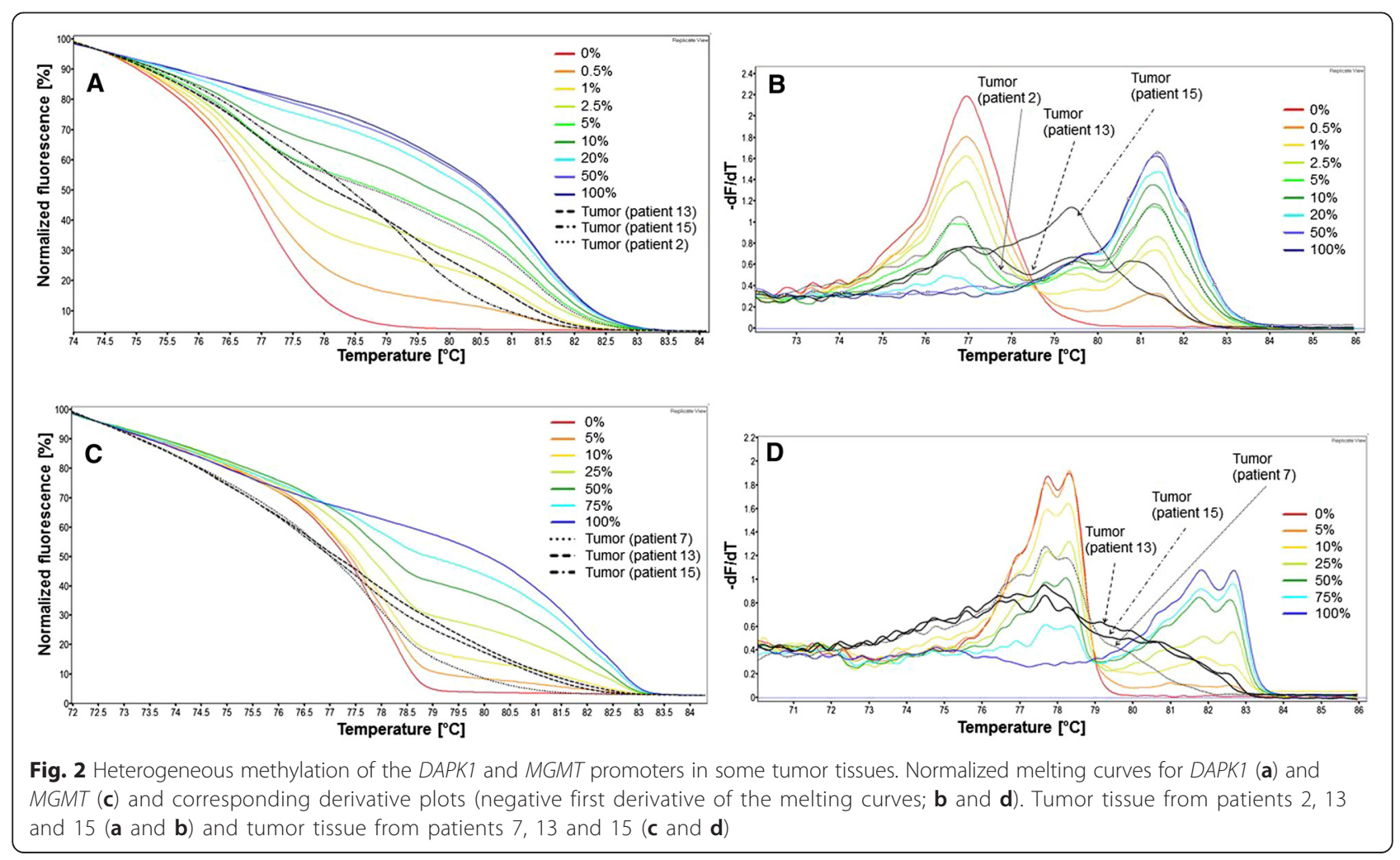


Methylation status in breast tissues of healthy controls DNA extracts from breast tissues of four healthy women that had been undergoing breast reduction mammoplasty were analyzed to determine DNA methylation base levels in noncancerous breast tissues. In all breast tissues of the control group, the methylation status of $D A P K 1, H I N-1$ and RASSF1A was < LOD. In three tissues, the methylation status of CCND2, GSTP1 and $M G M T$ was found to be $<$ LOD. In one tissue, the methylation status of GSTP1 was <LOQ. In the breast tissue of another woman, CCND2 and MGMT were found to be methylated (CCND2: < LOQ, MGMT: $5.1 \%)$. From two of the four women we obtained tissues from the left and right breast. In these women, no difference was found between the methylation status in the left and in the right breast. For none of the tumor suppressor genes did we find an association between the age of the women and the DNA methylation status.

\section{Methylation status in tumor, tumor-adjacent and tumor-distant tissue}

In spite of the low LOQs of the MS-HRM methods, a rather high proportion of the tumor tissues, tumoradjacent tissues (located about $1 \mathrm{~cm}$ from the tumor) and tumor-distant tissues (located $3 \mathrm{~cm}$ from the tumor tissue) was found to show methylation status $<$ LOQ. In several samples, the promoter was unmethylated (< LOD). Figure 3 shows the percentage of tumor, tumor-adjacent and tumor-distant tissues having methylation status $<$ LOD, $<$ LOQ and $\geq$ LOQ for each of the six tumor suppressor genes.
In tumor tissues, the promoters of five of the six genes were frequently methylated (methylation status $\geq$ LOD). A total of $94 \%(16 / 17), 82 \%(14 / 17), 65 \%(11 / 17), 63 \%$ $(10 / 16)$ and $53 \%(9 / 17)$ of the tumors showed promoter methylation of RASSF1A, HIN-1, MGMT, DAPK1, and GSTP1, respectively. The promoter of CCND2 was methylated in only $35 \%(6 / 17)$ of the tumors. In each of the 17 tumors, the promoter of at least one of the six genes was found to be methylated. Twelve tumors (71 \%) showed promoter methylation of $\geq 4$ genes. In $82 \%(14 / 17)$ of the tumors, the promoters of both RASSF $1 A$ and HIN-1 were found to be methylated. In general, the methylation status was, however, rather low. In only $59 \%(10 / 17), 53 \%(9 / 17), 41 \%$ (7/17), $38 \%$ $(6 / 16)$ and $35 \%(6 / 17)$ of the tumor samples, RASSF1A, HIN-1, MGMT, DAPK1 and CCND2 promoters showed methylation status $\geq$ LOQ. In none of the tumors, GSTP1 promoter methylation was $\geq$ LOQ. In $41 \%(7 / 17)$ of the tumors, both RASSF $1 A$ and $H I N-1$ promoters showed methylation status $\geq$ LOQ.

In tumor-adjacent and tumor-distant tissues, the RASSF1A promoter was as frequently and the $H I N-1$, $M G M T$ and GSTP1 promoters were almost as frequently methylated as in tumors. In only $38 \%(6 / 16)$ of the tumor-adjacent and tumor-distant tissues, the DAPK1 promoter was found to be methylated, compared to $63 \%(10 / 16)$ in tumors. CCND2 was not methylated in any of the tumor-adjacent or tumor-distant tissues.

Figure 3 indicates that the percentage of tumor-distant tissues showing methylation status of RASSF1A, MGMT or DAPK1 promoters $\geq$ LOQ was lower (6\%) than the percentage of tumor-adjacent tissues (29\% (5/17), $19 \%$

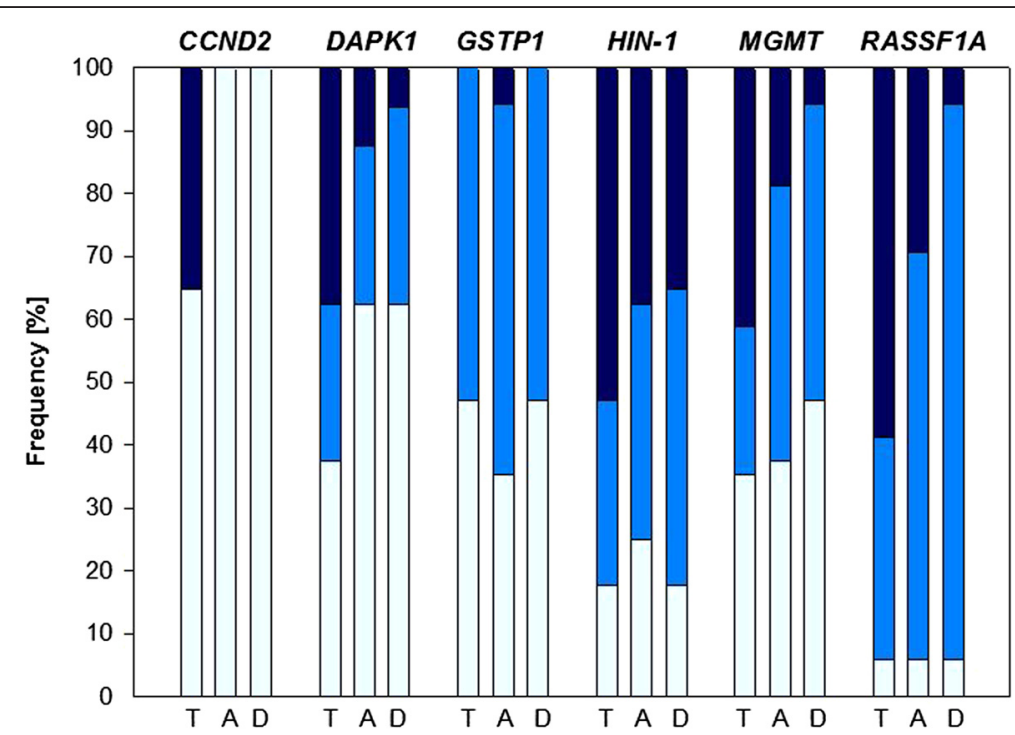

Fig. 3 Frequency of promoter methylation in tumor (T), tumor-adjacent $(A)$ and tumor-distant $(D)$ tissues. Light blue: methylation status $<$ LOD, middle blue: methylation status $<L O Q$, dark blue: methylation status $\geq L O Q$. LOD limit of detection, LOQ limit of quantification 
(3/16) and $13 \%(2 / 16)$, respectively), which, in turn, was lower than the percentage of tumor tissues (59\% (10/17), $41 \%(7 / 17)$ and $38 \%(6 / 16)$, respectively). With $35 \%(6 / 17)$ and $38 \%(6 / 16)$, the HIN-1 promoter rather frequently showed methylation $\geq$ LOQ in tumor-adjacent and tumor-distant tissues, respectively. In contrast, in only one of the tumor-adjacent tissues and none of the tumor-distant tissues, the methylation status of the GSTP1 promoter was $\geq \mathrm{LOQ}$.

Figure 4 illustrates the distribution of the promoter methylation levels in tumor, tumor-adjacent and tumor-distant tissues as well as in normal tissues of the control group. In order to be able to include methylation levels $<$ LOD and $<$ LOQ, values $<$ LOD and $<$ LOQ were substituted with default values, namely half the LOD and half the LOQ of the certain MS-HRM method, respectively. Figure 5 is limited to patients showing methylation status $\geq$ LOQ in the tumor tissue. As already discussed above, methylation levels were generally very low. In none of the samples did any of the genes show methylation status $>33 \%$. In only $18 \%(3 / 17)$ of the tumor tissues, the methylation status of HIN-1 and RASSF1A was $>20 \%$. The methylation status of CCND2 and DAPK1 was generally $<10 \%$. In five tumor tissues, none of the six genes showed a methylation status $\geq \mathrm{LOQ}$.

Statistically significant differences between the DNA methylation status in tumors and normal breast tissues of the control group were found for DAPK1 $(p=0.012)$, HIN-1 $(p=0.005)$ and RASSF1A $(p<0.001)$. Figure 4 indicates that in the case of MGMT, tumors showed a higher methylation status than normal breast tissues of the control group. Since in one of four women of the control group MGMT was methylated (methylation status $5.1 \%$ ), the difference was, however, not found to be statistically significant.

For all genes except GSTP1, statistically significant differences were found between the methylation status in tumor and tumor-distant tissues of breast cancer patients. This holds for all patients (Fig. 4) and the subgroup (methylation status of the certain gene promoter in the tumor $\geq \mathrm{LOQ}$, Fig. 5). The methylation status of RASSF1A, DAPK1 and CCND2 in tumor-adjacent tissues was significantly different from that in tumors. A statistically significant difference between the methylation status in tumor and tumor-adjacent tissues was also found for MGMT, but only for the subgroup (Fig. 5). In case of HIN-1, the methylation status in tumoradjacent tissues strongly correlated with that in tumors $(\mathrm{r}=0.785, p<0.001)$ (Fig. 6), but not with that in tumordistant tissues $(\mathrm{r}=0.312, p=0.239)$. For HIN-1, RASSF1A and MGMT, the methylation status in tumor-adjacent tissues was higher than that in tumor-distant tissues but the differences were not statistically significant.

\section{Association between methylation status and clinicopathological parameters}

When we investigated if the methylation status of the six tumor suppressor gene promoters is associated with clinicopathological characteristics (Table 1) of the breast cancer patients, we used the methylation status as categorical variable and divided the data set into two categories: "methylation status < LOD" and "methylation status > LOD".

The methylation status of HIN-1 in tumor tissues correlated with the age of the patients $(\mathrm{r}=0.555, p=0.021)$ (Fig. 7). In addition, we found association between the methylation status of $H I N-1$ in the adjacent tissues and the HER2 status $(p=0.039)$. In patients with HER2positive tumors, the methylation status of $H I N-1$ was more frequently<LOD than in patients with HER2negative status. The methylation status of MGMT in tumor-distant tissues was associated with tumor grading $(p=0.019)$. In patients with tumor grade 2 , the methylation status of MGMT in tumor-distant tissues was most frequently < LOD whereas in patients with tumor grade 3, the MGMT promoter was found to be methylated.

\section{Correlation between the methylation status of different genes}

We also evaluated if there is a statistically significant correlation between the promoter methylation status of different tumor suppressor genes in tumor tissues of one and the same patient. Correlation analyses revealed that the methylation status of RASSF1A positively correlated with that of $H I N-1 \quad(\mathrm{r}=0.600, p=0.011)$ (Fig. 8) and $\operatorname{MGMT}(\mathrm{r}=0.523, p=0.031)$. In addition, we found a positive correlation between promoter methylation of $D A P K 1$ and MGMT $(\mathrm{r}=0.514, p=0.042)$.

\section{Discussion}

We determined the methylation status of a panel of six tumor suppressor gene promoters in tumor, tumoradjacent and tumor-distant tissues of 17 breast cancer patients. CCND2, DAPK1, GSTP1, HIN-1, MGMT and RASSF1A were selected because they have previously been reported to be frequently methylated in primary breast cancers. Tumor-adjacent and tumor-distant tissues appeared histologically normal and were located about $1 \mathrm{~cm}$ and about $3 \mathrm{~cm}$ from the tumor, respectively. Normal breast tissues of women that had been undergoing reduction mammoplasty served as control.

The DNA methylation status was determined by MSHRM analysis, which consists of the following steps: treatment of the DNA with sodium bisulfite in order to convert unmethylated cytosines into uraciles (methylated cytosines remain unchanged); amplification of the bisulfite-treated DNA by PCR (uracils are replicated as thymines and methylated cytosines as cytosines); brief 


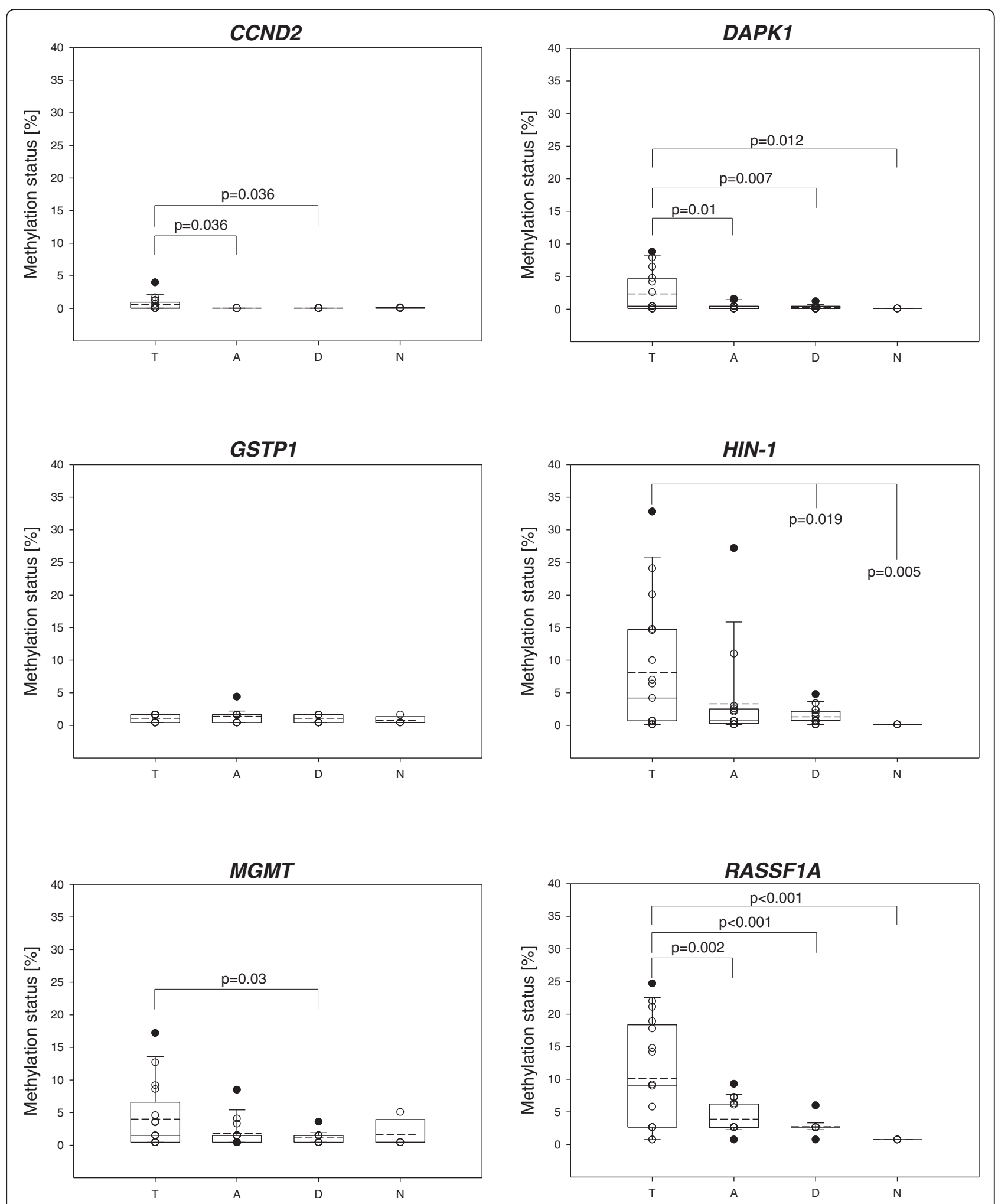

Fig. 4 Distribution of the methylation status in tumor (T), tumor-adjacent $(A)$ and tumor-distant $(D)$ tissues as well as normal breast tissues obtained from women undergoing reduction mammoplasty $(M)$. All patients have been included. Straight line: median, dashed line: arithmetic mean 

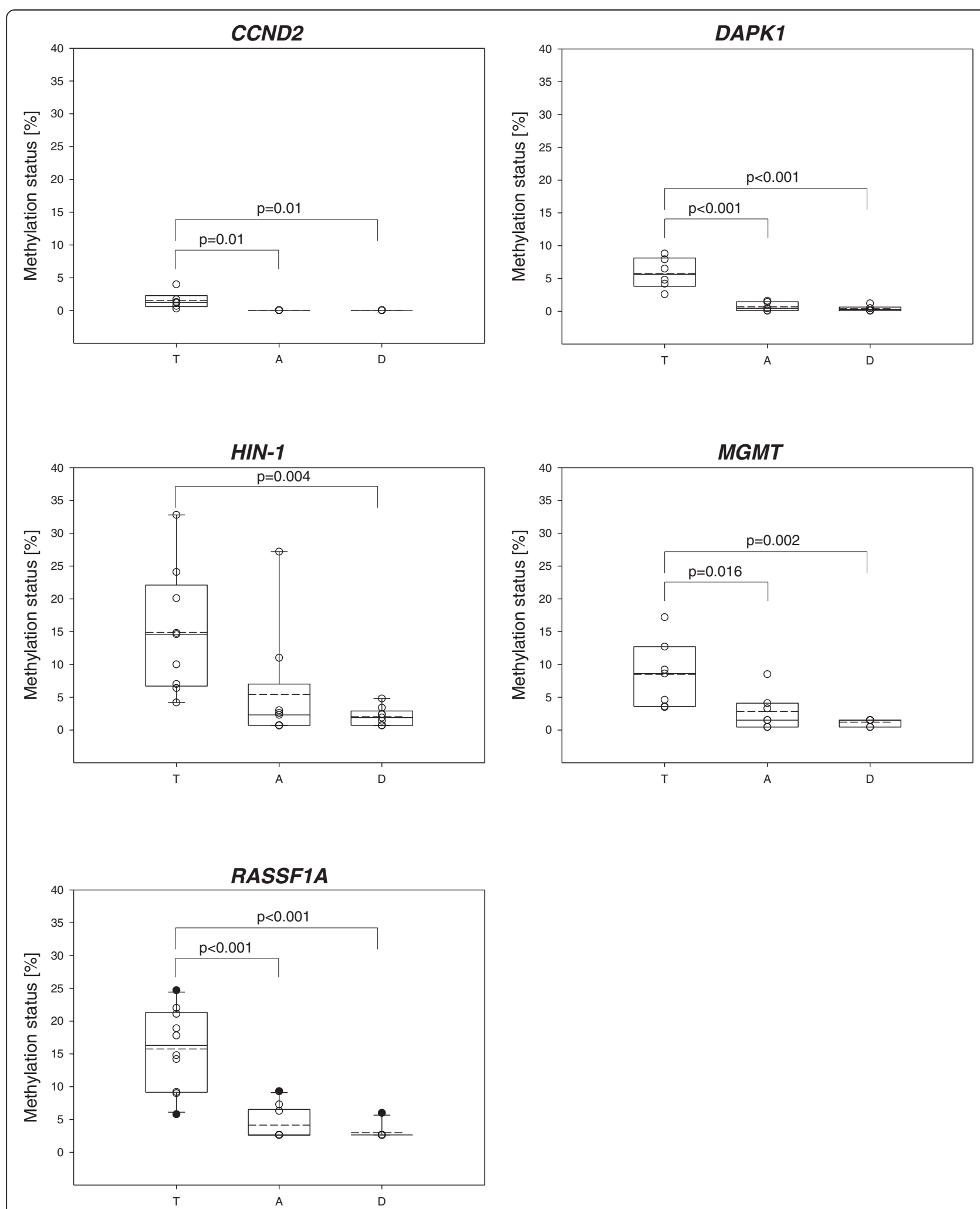

Fig. 5 Distribution of the methylation status in tumor $(T)$, tumor-adjacent $(A)$ and tumor-distant $(D)$ tissues. Patients have only been included if the methylation status in the tumor tissue was $\geq$ LOQ. Straight line: median, dashed line: arithmetic mean. LOQ limit of quantification 


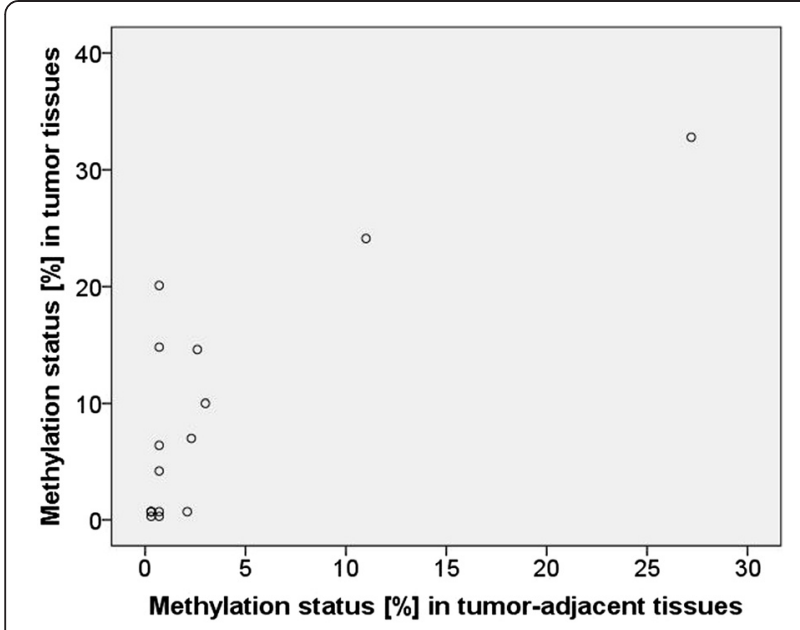

Fig. 6 Correlation of the methylation status of the HIN-1 promoter in tumor and tumor-adjacent tissues

denaturation and rapid reannealing of the PCR products; and finally high-resolution melting by gradually increasing the temperature. Guanine-cytosine-rich sequences (resulting from methylated $\mathrm{CpG}$ dinucleotides) melt at higher temperature than adenine-thymine-rich sequences (resulting from unmethylated CpG dinucleotides). Melting curves are obtained by plotting the fluorescence intensity against the temperature. The methylation status of unknown samples can be determined with the help of a calibration curve established by analyzing mixtures of unmethylated and fully methylated control DNA.

In our study MS-HRM analysis was the method of choice because we aimed at determining very low methylation levels and discriminating between small differences in the methylation status of the tumor suppressor gene promoters. MS-HRM methods allow adjusting

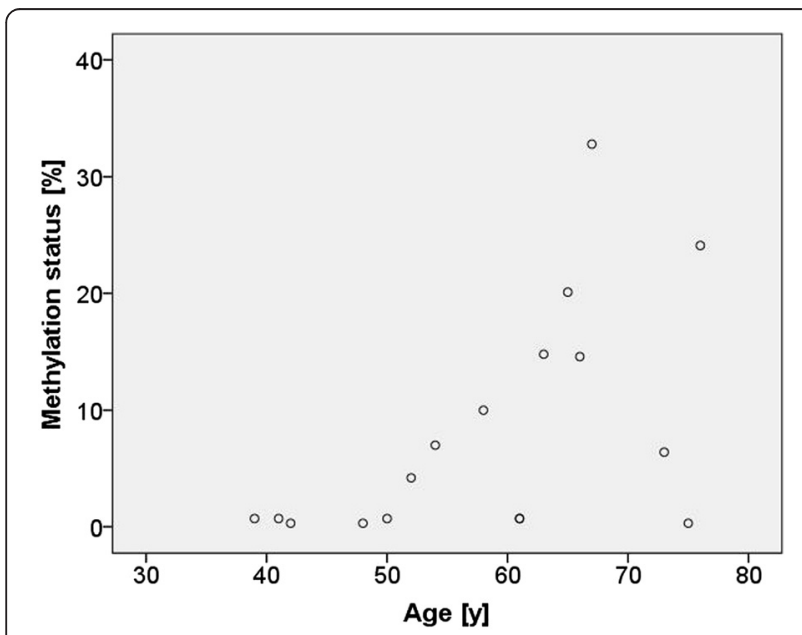

Fig. 7 Correlation of HIN-1 promoter methylation in tumor tissues with the age of the patients

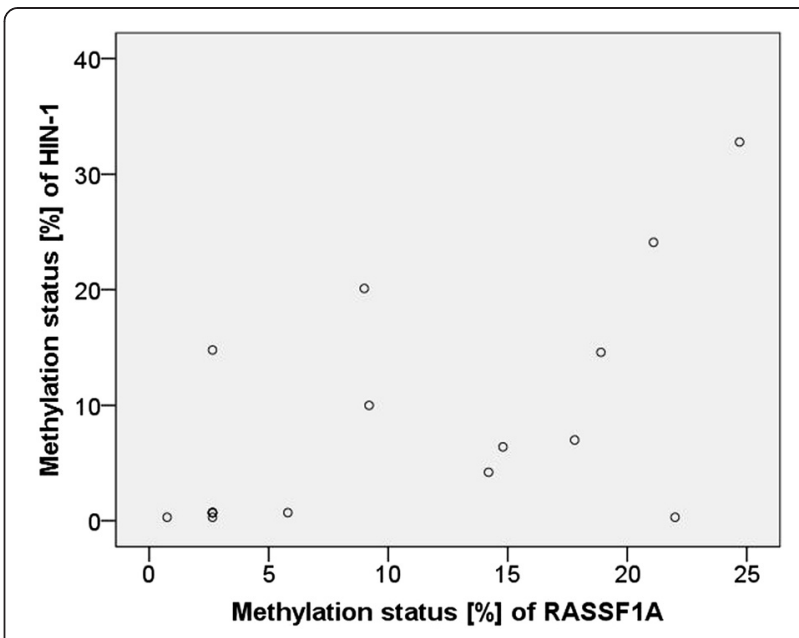

Fig. 8 Correlation of the methylation status of $\mathrm{HIN}^{-1}$ promoter and RASSFIA promoter in tumor tissues

the accessible methylation range by generating a (helpful) bias toward methylated or unmethylated alleles in PCR amplification [29]. High sensitivity can be achieved by using primers containing a low number of CpG dinucleotides, favoring amplification of guaninecytosine-rich sequences (resulting from methylated $\mathrm{CpG}$ dinucleotides) [23]. In addition, the $\mathrm{T}_{\mathrm{a}}$ and the $\mathrm{MgCl}_{2}$ concentration can be varied in order to tailor the methylation range accessible. LODs $(\mathrm{S} / \mathrm{N}=3)$ and LOQs $(\mathrm{S} / \mathrm{N}=10)$ of the MS-HRM methods applied were determined by repeatedly analyzing bisulfite-treated, unmethylated control DNA. The methods proved to be very sensitive, with LODs and LOQs ranging from 0.1 to $1.5 \%$ and 0.3 to $5.3 \%$, respectively.

In contrast to several other methods used in DNA methylation analysis, e.g., bisulfite pyrosequencing, MSHRM methods do not allow determining the methylation status of individual $\mathrm{CpG}$ dinucleotides. From the melting profiles and the corresponding derivative plots one can, however, assess if the original template was methylated homogeneously or heterogeneously. In the reannealing step, carried out after briefly denaturing the PCR products, DNA sequences originating from homogenously methylated templates form homoduplexes, whereas those originating from partially methylated templates form heteroduplexes. Due to base-pairing mismatches, heteroduplexes begin to melt at lower temperature and lead to more complex melting profiles than homoduplexes. In biopsy samples analyzed in the present study, promoters of CCND2, GSTP1, HIN-1 and RASSF1A were found to be methylated homogenously (in case they were methylated at all). Most of the tumors showed homogenous methylation of DAPK1 and $M G M T$, in some tumor tissues the promoter of these genes was methylated heterogeneously. We did not find 
an association between the occurrence of heterogeneous methylation in DAPK1 and MGMT promoters and any of the clinicopathological parameters of the patients. Heterogeneous methylation of DAPK1 promoter has been shown previously in patients with chronic lymphocytic leukemia [30] or diffuse large B-cell lymphoma [31]. The MGMT promoter has been found to be methylated heterogeneously in patients with breast cancer [24], in diffuse large B-cell lymphoma [31] and in human breast cancer cell line HS578T [32].

In MS-HRM analysis, the DNA methylation status is determined with the help of calibration curves established by analyzing mixtures of unmethylated and methylated human control DNA. In case of heterogeneous methylation, melting curves differ in shape from melting curves obtained for standards. In order to obtain accurate results in spite of differences in the shape of the melting curves, we established interpolation calibration curves by taking into account the average of the normalized fluorescence signal for each standard over the entire temperature.

None of the normal breast tissues of the control group showed methylation of DAPK1, HIN-1 and RASSF1A. In one tissue, the methylation status of GSTP1 was $<\mathrm{LOQ}$. In the breast tissue of the oldest woman (age: 60 years), the promoters of CCND2 and MGMT were found to be methylated (CCND2 L LOQ, MGMT: $5.2 \%)$. However, for none of the tumor suppressor genes did we find an association between the age of the women and the DNA methylation status.

In breast cancer patients, $94 \%$ and $82 \%$ of the tumor tissues showed methylation of RASSF1A and HIN-1 promoters, respectively. In $82 \%$ of the tumors, even both promoters were methylated. Our results are in accordance with previous studies reporting frequent methylation of RASSF1A and/or HIN-1 in breast carcinoma. RASSF1A methylation was found in $85 \%$ [33-35], $68 \%$ [36], $65 \%$ [37], $59 \%$ [38], $58 \%$ [21] and $33 \%$ [24] of the breast tumors analyzed, HIN-1 in $74 \%$ [39]), $73 \%$ [33] and $49 \%$ [21] of the cases. In the present study, promoters of MGMT, DAPK1 and GSTP1 were methylated in $65 \%, 63 \%$ and $53 \%$ of the tumors. However, in case of GSTP1, all tumors showed methylation status $<\mathrm{LOQ}$. Frequency of methylation of these promoters was higher than reported in the literature (MGMT: $22 \%$ [24]; DAPK1: $50 \%$ [37] and $37.5 \%$ [24]; GSTP1: $16.6 \%$ [24] and $14 \%$ [40]), most probably due to the high sensitivity (LOD $<1 \%$ ) of the MS-HRM methods applied in the present study. In contrast, the CCND2 promoter was found to be less frequently methylated (in $35 \%$ of the tumors) than in the study of Lewis et al. (57 \% [38]).

In each tumor tissue, the promoter of at least one of the six genes was found to be methylated. A total of $71 \%$ of the tumors showed promoter methylation of $\geq 4$ genes. This finding is consistent with previous studies reporting methylation of more than one tumor suppressor gene in breast tumors [24, 37]. Simultaneous methylation of several tumor suppressor genes indicates the important role DNA methylation is playing in breast cancer development.

In tumors, the DNA methylation status of DAPK1 $(p=0.012)$, HIN-1 $(p=0.005)$ and RASSF1A $(p<0.001)$ was statistically significantly different from that in normal breast tissues of the control group. In addition, tumor tissues showed a higher methylation status of MGMT (Fig. 4) compared to the control group, however, the difference was not statistically significant since one normal breast tissue had a methylation status of $5.1 \%$ (other normal breast tissues of the control group had a methylation status $<$ LOD). In the present study, MGMT promoter methylation was detected in the oldest woman of the control group. In a previous study, MGMT methylation has been associated with the age of breast cancer patients [24]. However, in our study we did not find a significant correlation between the DNA methylation status of MGMT and the age of the women, neither for breast cancer patients nor for the control group.

With the exception of CCND2 and DAPK1, the genes were not only frequently methylated in the tumors but also in the corresponding tumor-adjacent and tumordistant tissues of the breast cancer patients. The RASSF1A promoter was as frequently and the HIN-1, $M G M T$ and GSTP1 promoters were almost as frequently methylated as in tumors. With the exception of GSTP1, the methylation status was significantly lower in tumordistant tissues than in tumors. In case of RASSF1A, $H I N-1$ and MGMT promoters, tumor-adjacent tissues showed higher methylation status than tumor-distant tissues, the difference was, however, not statistically significant. These results demonstrate that the methylation status of RASSF1A, HIN-1 and MGMT promoters indicates field cancerization in breast cancers. To the best of our knowledge, field cancerization due to MGMT promoter methylation has not been reported so far. MGMT field cancerization has, however, been detected in colorectal cancers $[14,41]$ and oral squamous cell carcinomas [42]. Aberrant methylation in tissue adjacent to breast tumor has already been reported previously for RASSF1A [20, 21] and HIN-1 [21]. Methylation of the $H I N-1$ promoter has been detected with high frequency (95\%) in preinvasive lesions such as ductal and lobular carcinoma in situ [39], indicating that it is an early event in breast tumorigenesis. In the present study, the methylation status of the $H I N-1$ promoter in tumor-adjacent tissues was found to correlate strongly with that in the corresponding tumors, but not with that in the corresponding tumor-distant tissues. Among the promoters investigated in the present study, the methylation status 
of the HIN-1 promoter can thus be considered the best suitable biomarker for detecting field cancerization. Further investigation is needed to test whether it can be used for defining surgical margins in order to prevent future recurrence of breast cancer.

Breast cancer is known to be a heterogeneous disease with regard to histopathological and molecular characteristics, outcome and response to treatment. Previous studies have reported association between the methylation status of a variety of gene promoters and clinicopathological parameters.

$H I N-1$ is a putative cytokine reported to be highly expressed in normal but not cancerous mammary epithelial cells $[39,43]$. The methylation status of HIN-1 in breast tumors has been associated with the ER, PR and/ or HER2 status [21, 43]. In our study, in tumor-adjacent tissues of patients with HER2-positive tumors the HIN-1 promoter was more frequently unmethylated (< LOD) than in those from patients with HER2-negative status. In addition, we found association between the methylation status of $H I N-1$ in the tumor-distant tissue and the PR and ER status of the tumor. However, the number of PR-negative and ER-negative tumors investigated was too low to allow a statistical conclusion. The methylation status of the HIN-1 promoter in tumors was found to correlate with the age of the patients, which is in accordance with the study of Feng et al. [21].

MGMT plays a role in DNA repair. Silencing of MGMT by promoter methylation is a predictor of overall survival and response to alkylating agents [44]. In our study, the methylation status of MGMT in tumor-distant tissues was associated with tumor grading. In patients with tumor grade 3, the MGMT promoter was found to be more frequently methylated than in patients with tumor grade 2. In the study of Tserga et al. [24], the methylation status of the MGMT promoter in the tumor itself was found to be associated with advanced tumor grade.

Several studies report association between RASSF1A promoter methylation and ER status of the tumor $[35,45,46]$. In the present study only one of the tumors was ER-negative. We therefore could not evaluate potential association between the methylation status of the RASSF1A promoter and the ER status of the tumors.

As already mentioned above, more than one tumor suppressor gene has been found to be methylated in a high percentage of the tumors. Correlation analyses revealed that the methylation status of RASSF1A positively correlated with that of $H I N-1$ and MGMT. In addition, we found a statistically significant positive correlation between the promoter methylation of DAPK1 und $M G M T$. Correlation between methylation levels of RASSF $1 A$ and HIN-1 in tumor of breast cancer patients has been previously published by Feng et al. [21].

\section{Conclusions}

MS-HRM analysis was carried out to determine the methylation status of CCND2, DAPK1, GSTP1, HIN-1, $M G M T$ and RASSF1A promoters in tumor tissues and histologically normal-appearing tumor-adjacent and tumor-distant tissues of 17 breast cancer patients. More than half of the tumors showed promoter methylation of RASSF1A, HIN-1, MGMT, DAPK1 and GSTP1. In a high percentage of the tumors, more than one gene promoter was methylated. The methylation status of the HIN-1 promoter was found to correlate with the age of the patients. Not only the tumors but also the corresponding tumor-adjacent and tumor-distant tissues frequently showed DNA methylation. The methylation status of the RASSF1A, HIN-1 and MGMT promoters was significantly lower in tumor-distant tissues than in tumors. In tumor-adjacent tissues, the methylation status of these gene promoters was found to be higher than in tumordistant tissues, indicating field cancerization. So far, field cancerization due to MGMT promoter methylation has been reported for colorectal cancers and oral squamous cell carcinomas, but not for breast cancer. In the case of the HIN-1 promoter, the methylation status in tumoradjacent tissues correlated strongly with that in the corresponding tumor tissues. Among the promoters investigated, HIN-1 promoter methylation can therefore be considered the best suitable biomarker for detecting field cancerization. Further studies should be carried out to investigate its applicability for defining surgical margins in order to prevent future recurrence of breast cancer.

\section{Abbreviations}

CCND2: cyclin D2; CDH13: cadherin-13; CpG: cytosine-phosphatidyl-guanosine; DAPK1: death-associated protein kinase 1; ER: estrogen receptor;

GSTP1: glutathione S-transferase pi 1; IDC: invasive ductal carcinoma; ILC: invasive lobular carcinoma; HER2: human epidermal growth factor receptor 2; HIN-1: high in normal 1; LOD: limit of detection; LOQ: limit of quantification; MGMT: 0-6-methylguanine-DNA methyltransferase; MIB-1: mindbomb E3 ubiquitin protein ligase 1; MS-HRM: methylation-sensitive high-resolution melting; PBS: phosphate-buffered saline; PCR: polymerase chain reaction; PR: progesterone receptor; RASSF1A: Ras association domain family member 1; RIL: reversion-induced LIM protein; S/N: signal-to-noise ratio; $\mathrm{T}_{\mathrm{a}}$ : annealing temperature.

\section{Competing interests}

The authors declare that they have no competing interests.

\section{Authors' contributions}

MS performed experiments, analyzed data, prepared figures and tables and performed statistical analyses. EH performed experiments and analyzed data. GP and SH carried out tissue procurement and patient data collection. MCM supported conception and design and drafted the manuscript. All authors read and approved the final manuscript.

\section{Author details}

'Department of Analytical Chemistry, University of Vienna, Währinger Str. 38, 1090 Vienna, Austria. ${ }^{2}$ Department of Obstetrics and Gynecology, Division of Gynecology and Gynecological Oncology, Medical University of Vienna, Währinger Gürtel 18-20, 1090 Vienna, Austria. ${ }^{3}$ Department of Plastic and Reconstructive Surgery, Medical University of Vienna, Währinger Gürtel 18-20, 1090 Vienna, Austria. 
Received: 6 November 2014 Accepted: 27 August 2015

\section{Published online: 14 September 2015}

\section{References}

1. Chin L, Andersen JN, Futreal PA. Cancer genomics: from discovery science to personalized medicine. Nat Med. 2011;17:297-303.

2. Simó-Riudalbas L, Esteller M. Cancer genomics identifies disrupted epigenetic genes. Hum Genet. 2014;133:713-25.

3. Tsai HC, Baylin SB. Cancer epigenetics: linking basic biology to clinical medicine. Cell Res. 2011;21:502-17.

4. Berdasco M, Esteller M. Aberrant epigenetic landscape in cancer: how cellular identity goes awry. Dev Cell. 2010;19:698-711.

5. Brooks J, Cairns P, Zeleniuch-Jacquotte A. Promoter methylation and the detection of breast cancer. CCC. 2009;20:1539-50.

6. Delpu Y, Cordelier P, Cho WC, Torrisani J. DNA methylation and cancer diagnosis. Int J Mol Sci. 2013;14:15029-58.

7. Garcia-Gimenez JL, Sanchis-Gomar F, Lippi G, Mena S, Ivars D, Gomez-Cabrera MC, et al. Epigenetic biomarkers: A new perspective in laboratory diagnostics. Clin Chim Acta. 2012;413:1576-82.

8. Szyf M. DNA methylation signatures for breast cancer classification and prognosis. Genome Med. 2012:4:26.

9. Duffy MJ, Napieralski R, Martens JW, Span PN, Spyratos F, Sweep FC, et al. Methylated genes as new cancer biomarkers. Eur J Cancer. 2009;45:335-46.

10. Duffy MJ. Tumor markers in clinical practice: a review focusing on common solid cancers. Med Princ Pract. 2013;22:4-11.

11. Egger G, Liang G, Aparicio A, Jones PA. Epigenetics in human disease and prospects for epigenetic therapy. Nature. 2004;429:457-63.

12. Cai FF, Kohler C, Zhang B, Wang MH, Chen WJ, Zhong XY. Epigenetic therapy for breast cancer. Int J Mol Sci. 2011;12:4465-87.

13. Chai H, Brown RE. Field effect in cancer-an update. Ann Clin Lab Sci. 2009:39:331-7.

14. Shen L, Kondo Y, Rosner GL, Xiao L, Hernandez NS, Vilaythong J, et al. MGMT promoter methylation and field defect in sporadic colorectal cancer. J Natl Cancer Inst. 2005;97:1330-8.

15. Belshaw NJ, Elliott GO, Foxall RJ, Dainty JR, Pal N, Coupe A, et al. Profiling $\mathrm{CpG}$ island field methylation in both morphologically normal and neoplastic human colonic mucosa. Br J Cancer. 2008:99:136-42.

16. Subramaniam MM, Loh M, Chan JY, Liem N, Lim PL, Peng YW, et al. The topography of DNA methylation in the non-neoplastic colonic mucosa surrounding colorectal cancers. Mol Carcinogen. 2014;53:98-108.

17. Mehrotra J, Varde S, Wang H, Chiu H, Vargo J, Gray K, et al. Quantitative, spatial resolution of the epigenetic field effect in prostate cancer. Prostate. 2008;68:152-60.

18. Yang B, Bhusari S, Kueck J, Weeratunga P, Wagner J, Leverson G, et al. Methylation profiling defines an extensive field defect in histologically normal prostate tissues associated with prostate cancer. Neoplasia. 2013;15:399-408

19. Dakubo GD, Jakupciak JP, Birch-Machin MA, Parr RL. Clinical implications and utility of field cancerization. Cancer Cell Int. 2007;7:2.

20. Yan PS, Venkataramu C, Ibrahim A, Liu JC, Shen RZ, Diaz NM, et al. Mapping geographic zones of cancer risk with epigenetic biomarkers in normal breast tissue. Clin Cancer Res. 2006;12:6626-36.

21. Feng W, Shen L, Wen S, Rosen DG, Jelinek J, Hu X, et al. Correlation between $\mathrm{CpG}$ methylation profiles and hormone receptor status in breast cancers. Breast Cancer Res. 2007;9:R57.

22. Jovanovic J, Ronneberg JA, Tost J, Kristensen V. The epigenetics of breast cancer. Mol Oncol. 2010:4:242-54.

23. Wojdacz TK, Borgbo T, Hansen LL. Primer design versus PCR bias in methylation independent PCR amplifications. Epigenetics. 2009:4:231-4.

24. Tserga A, Michalopoulos NV, Levidou G, Korkolopoulou P, Zografos G,

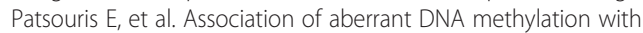
clinicopathological features in breast cancer. Oncol Rep. 2012;27:1630-8.

25. Avraham A, Uhlmann R, Shperber A, Birnbaum M, Sandbank J, Sella A, et al. Serum DNA methylation for monitoring response to neoadjuvant chemotherapy in breast cancer patients. Int J Cancer. 2012;131:E1166-72.

26. Migheli F, Stoccoro A, Coppedè F, Wan Omar WA, Failli A, Consolini R, et al. Comparison study of MS-HRM and pyrosequencing techniques for quantification of APC and CDKN2A gene methylation. PLOS One. 2013;8:e52501.

27. Office of Pesticide Programs, U.S. Environmental Protection Agency Washington, DC: Assigning values to non-detected/non-quantified pesticide residues in human health food exposure assessments. March 23, 2000. http://www.epa.gov/oppfead1/trac/science/trac3b012.pdf. October 30th 2014.

28. Candiloro IL, Mikeska T, Hokland P, Dobrovic A. Rapid analysis of heterogeneously methylated DNA using digital methylation-sensitive high resolution melting: application to the CDKN2B (p15) gene. Epigenetics Chromatin. 2008:1:7.

29. Wojdacz TK, Moller TH, Thestrup BB, Kristensen LS, Hansen LL. Limitations and advantages of MS-HRM and bisulfite sequencing for single locus methylation studies. Expert Rev Mol Diagn. 2010;10:575-80.

30. Candiloro ILM, Mikeska T, Dobrovic A. Assessing combined methylationsensitive high resolution melting and pyrosequencing for the analysis of heterogeneous DNA methylation. Epigenetics. 2011;6:500-7.

31. Kristensen LS, Treppendahl MB, Asmar F, Girkov MS, Nielsen HM, Kjeldsen TE, et al. Investigation of MGMT and DAPK1 methylation patterns in diffuse large B-cell lymphoma using allelic MSP-pyrosequencing. Sci Rep. 2013;3:2789.

32. Wojdacz TK, Dobrovic A. Methylation-sensitive high resolution melting (MS-HRM): a new approach for sensitive and high-throughput assessment of methylation. Nucleic Acids Res. 2007:35:e41.

33. Bae YK, Brown A, Garrett E, Bornman D, Fackler MJ, Sukumar S, et al. Hypermethylation in histologically distinct classes of breast cancer. Clin Cancer Res. 2004:10:5998-6005.

34. Shukla S, Mirza S, Sharma G, Parshad R, Gupta SD, Ralhan R. Detection of RASSFTA and RARB hypermethylation in serum DNA from breast cancer patients. Epigenetics. 2006;1:88-93.

35. Klajic J, Fleischer T, Dejeux E, Edvardsen H, Warnberg F, Bukholm I, et al. Quantitative DNA methylation analyses reveal stage dependent DNA methylation and association to clinico-pathological factors in breast tumors. BMC Cancer. 2013;13:456.

36. Yan PS, Shi H, Rahmatpanah F, Hsiau TH, Hsiau AH, Leu YW, et al, Differential distribution of DNA methylation within the RASSF1A CpG island in breast cancer. Cancer Res. 2003;63:6178-86.

37. Dulaimi E, Hillinck J, Ibanez De Caceres I, Al-Saleem T, Cairns P. Tumor suppressor gene promoter hypermethylation in serum of breast cancer patients. Clin Cancer Res. 2004:10:6189-93.

38. Lewis CM, Cler LR, Bu DW, Zöchbauer-Müller S, Milchgrub S, Naftalis EZ, et al. Promoter hypermethylation in benign breast epithelium in relation to predicted breast cancer risk. Clin Cancer Res. 2005;11:166-72.

39. Krop IE, Sgroi D, Porter DA, Lunetta KL, LeVangie R, Seth P, et al. HIN-1, a putative cytokine highly expressed in normal but not cancerous mammary epithelial cells. Proc Natl Acad Sci U S A. 2001;98:9796-801.

40. Arai T, Miyoshi Y, Kim SJ, Taguchi T, Tamaki Y, Noguchi S. Association of GSTP1 CpG islands hypermethylation with poor prognosis in human breast cancers. Breast Cancer Res Treat 2006;100:169-76.

41. Farzanehfar M, Vossoughinia H, Jabini R, Tavassoli A, Saadatnia $H$, Khorashad AK, et al. Evaluation of methylation of MGMT (O(6)methylguanine-DNA methyltransferase) gene promoter in sporadic colorectal cancer. DNA Cell Biol. 2013:32:371-7.

42. Supic G, Kozomara R, Jovic N, Zeljic K, Magic Z. Prognostic significance of tumor-related genes hypermethylation detected in cancer-free surgical margins of oral squamous cell carcinomas. Oral Oncol. 2011;47:702-8.

43. Krop I, Maguire P, Lahti-Domenici J, Lodeiro G, Richardson A, Johannsdottir HK, et al. Lack of HIN-1 methylation in BRCA1-linked and "BRCA1-like" breast tumors. Cancer Res. 2003;63:2024-7.

44. Gerson SL. MGMT: its role in cancer aetiology and cancer therapeutics. Nat Rev Cancer. 2004;4:296-307.

45. Sunami E, Shinozaki M, Sim MS, Nquyen SL, Vu AT, Giuliano AE, et al. Estrogen receptor and HER2/neu status affect epigenetic differences of tumor-related genes in primary breast tumors. Breast Cancer Res. 2008;10:R46

46. Kajabova V, Smolkova B, Zmetakova I, Sebova K, Krivulcik T, Bella V, et al. RASSFIA promoter methylation levels positively correlate with estrogen receptor expression in breast cancer patients. Transl Oncol. 2013;6:297-304. 